\title{
Effects of Academic Stress on Teenagers with Type 1 Diabetes
}

\author{
Britney Perez ${ }^{1}$ and Jannette Sierra\# \\ ${ }^{1}$ Pinecrest Preparatory High School, Miami, FL, USA \\ \#Advisor
}

$\underline{\text { ABSTRACT }}$

Type one diabetes is a a condition that is exponentially growing in the US and all over the world. As the majoirty of these patients are diagnosed at a young age, much research is being completed in order to find ways to help these adolescents live a more normal life by minimizing its effects. The goal of this research is to find a possible correlation between diabetic teenagers' academic stress levels and their average glucose levels. By means of an extensive 3 week survey in which each participant was asked to share their stress levels, lifestyle habits, and glucose reports on a daily basis, it was found that there is a strong positive correlation between he two variables. They produced an r-value of about 0.9939 , suggesting statistical significance. Knowing this information will help patients within this age group manage their glucose readings better and keep them at better control throughout the day.

\section{Literature Review}

For the purposes of understanding this study, it is critical to analyze and understand the works and findings previously found within the realm of this topic. According to the American Diabetes Association (ADA, 2018), about 1.6 million people in the United States have type one diabetes and of that number, 187,000 are under the age of 20. A prevalent issue within minors, especially in their teenage years, is dealing with great amounts of stress coming from various aspects of their life. Despite its magnitude, we know very little about how it affects glycemic levels in type one diabetics. Through numerous medical studies and trials conducted on the autoimmune disease we do know that hyperglycemia, high blood sugar, causes stress on the body, such as cytokine and metabolic stress. (Bending, et al., 2012) This phenomenon can occur in both diabetic and non-diabetic patients. It can also lead to weight loss, fatigue, and poor blood circulation, early signs of someone diagnosed with diabetes. However, we are still left with the opposite question: hyperglycemia has been established as causing stress, but does it work in reverse? Does stress cause abnormal glucose levels?

Researchers actually have reached some conclusions for this question, but unfortunately not pertaining to humans. Research has been performed on lab rats in various studies in the past and at least one of the studies has even used crabs to examine their glycemic levels caused by stress (Trapp, et al., 2018). In a study performed in the early 1990's (Surwit, et al., 1992) as well as one performed more recently (Radahmadi, et al., 2006), groups of type one diabetic rats were put under stressful conditions and then had their blood sugar measured periodically. Results from both studies show that, following the stressful conditions, there was an exponential increase of blood sugar which ultimately led to hyperglycemia in the rats. In the latter study, non-diabetic rats were also used and had the same outcome. They also noted that there appeared to be a significant weight loss more commonly in the diabetic rats, which as previously mentioned, is a symptom in human hyperglycemic patients. Research studying the glycemic levels of crabs, specifically the euryhaline crab Neohelice granulata, in relation to stress, similarly concluded that stress affects a process in crabs closely resembling insulin signaling and causes their glucose uptake in their posterior gills to increase, ultimately creating hyperglycemia (Trapp, et al., 2018). They came to this conclusion by submitting the crabs to three periods of hyperosmotic and hyposmotic stress for 24, 72, and 144 hours and followed up by 
investigating the insulin-like receptor phosphorylation capacity of their gills. (Trapp, et al., 2018) Although all three of these studies performed show a clear positive correlation between physiological stress and glycemic levels, they only pertain to animals and not specifically humans. It can be inferred that the same will likely happen with humans, but there is no definitive evidence and since the human body system differs from those of animals and vary in function, animal results may not be fully generalizable to humans. Although there is limited human research directly focused on stress as a cause of hyperglycemia, many other research has explored both factors, stress and glucose levels, together through different questions. A study released by "The Journal of Diabetes Research" in August of 2020 does show a negative impact of stress on self-managed diabetes, but with a much different approach than does this particular study. Instead of finding a direct correlation between stress and glycemic levels, which is the ultimate goal of this study, they connected stress to dietary habits and then to glycemic levels in patients of 40 years or younger (Ahola, et al., 2020). Using self-reported data along with a professional lab, perceived stress was measured on a numerical scale and then compared to dietary intake, glucose levels, and physical activity. They found that stressful situations and the feeling of stress in general causes people to eat more often in efforts to lower their stress levels, but by doing so, they are essentially causing their glucose levels to rise, especially if not taken care of properly by giving boluses of insulin for every meal or snack with carbohydrates. In another study, analyzing the relationship between stress and glucose levels through a different approach, type one diabetics between the ages of 16-30 were put into a stress management training program to evaluate its effects on glycemic levels (Attari, et al., 2006). Attari et al. did so by putting their group of participants through a three-month long stress-management training program and measuring their A1C levels before and after the training and then comparing the two. Their results support the idea that these training groups do significantly help diabetic patients maintain a better control of their readings and lower their A1C levels in a short period of time.

The previous studies discussed have been based solely on type one diabetes, as this particular study is, but there is also significant research of type 2 diabetics' glucose levels in relation to stress that might help get a better understanding for the overall topic. Goetsch et al., (1990), for example, became the model for this study, but used a group of type two diabetics, was a smaller sample, and focused on adults: "six adults with non-insulin dependent diabetes mellitus participated...[and]completed 12 days home monitoring of stressful events, subjective stress, and blood glucose" (Goetsch, et al., 1990, p. 2). The results proved that "blood glucose range tended to be greater on high vs low stress days" and "stress has a hyperglycemic effect on blood glucose" (Goetsch, et al., 1990, p. 2). In a more recent research study, lab rats were used to study the effects of oxidative stress on glucose levels, much like the studies previously mentioned, except that these rats were type two instead of one (Ferreira et al., 2010). The rats were put through stress-inducing scenarios for a period of 6 weeks and then had their glucose levels compared to that of before the stressful conditions. The results showed that "oxidative stress play[s] a major role in type 2 diabetes mellitus (T2DM) pathophysiology, contributing for obesity, insulin resistance and cardiovascular complications, which further aggravate the disease" (Ferreira et al., 2010, p.2). In each of these studies, stress was once again found to cause hyperglycemia by causing insulin resistance in the rat population.

By utilizing the results, methods, and more of these studies discussed, the study presented can be viewed from a much more broad and understanding perspective to have knowledge in the background of the topic of stress on diabetes. This information can also be used to an advantage by creating a study which has never previously been performed such as focusing on academic stress specifically as well as a specific teenage age group for only diabetics who are type one. Additionally, no other studies have utilized the tools provided by the Dexcom Continuous Glucose Monitor (CGM) which will be used in this one. Utilizing these components, this study addresses the question "What effects do academic stress levels have on the glycemic levels of type one diabetics between the ages of 14 and 18"?

\section{Methods}

A correlational study was used to answer the research question based on quantitative data from a survey. 
A survey was chosen as the ideal primary instrument because it allowed national reach, a larger sample, and less bureaucratic obstacles that come with a more experimental approach. This study focused on a sample group of 22 type one diabetics within the age range of 14 to 18 years old from different diabetes support groups across the United States. In regards to this study, they will be referred to as participant letters A-V in order to ensure their privacy and confidentiality. Additionally, the participants were required to have the Dexcom Continuous Glucose Monitor (CGM), so the researcher could easily track their average blood glucose levels throughout the week. Both males and females were included in this study to increase the generalizability of the results while also focusing on the unique gap addressed: academic stress specific, the age group of 14-18, and the Dexcom device.

Several platforms were utilzed for collecting data in this study. The first was a Google Form sent via email to get a participant and legal guardian signature in acknowledgment to the terms and conditions of the research. A Google Document with the survey containing questions on stress, exercise, and eating patterns was also individually sent out to each participant via email at the beginning of the survey period. After 3 weeks passed, the forms were then sent back to me fully completed in order to evaluate. Additionally, the Dexcom sharing feature through Dexcom Clarity was used to monitor the participants' weekly blood sugar results. The report sent included trend graphs, average glucose readings, and many extraneous factors. These graphs can be modified to show only a certain range of glucose reading numbers and also can be broken down into only certain days of the week or a certain time frame, for example a period of 14 days, or in the case of this study, for 21 days. (Dexcom Inc., 2021)

The data from this study was then collected from a survey done on a Google Document and from the weekly glucose reports from Dexcom. The survey contained three charts in which participants were asked to fill out in as much detail as they possibly could: the three charts were based on daily academic stress, daily exercise, and daily diet and insulin intake. As for academic stress, participants filled out how stressed they were feeling based on a 1-10 scale each day for a time period of three weeks. In order to determine this numerical value on their own, they wre asked to reflect on about how many hours were spent completeing homework, studying, or participating in other school based extracurricular activities as well as reflect on their daily overall mood and mental health. These questions were used to guide the participants, not make them determine a definitive stress score, as time and mood usually tend to play a When interpreting the scale, 1-3 indicated very little to no stress coming from academic work and activities for that day, 4-6 indicated a moderate level of stress coming from academic work and activities for that day, and 7-10 indicated a high level of stress coming from academic work and activities for that day. Moreover, in order to take into consideration confounding variables such as exercise, participants filled into the chart any physical activity they performed throughout the day, at approximately what time, and how strenuous they would rate it on a scale of 1-10. This scale was similarly weighed as the previous one in which 1-3 indicated very light physical activity performed during a provided time period, 4-6 indicated a moderate level of physical activity performed during a provided time period, and 7-10 indicated a high level of physical activity performed during a provided time period. This was also done everyday for a time period of three weeks. Finally, to take into consideration another confounding variable, diet and insulin boluses, participants were required to fill in a chart stating every time they had any sort of carbohydrate intake including the time, grams of carbs, and units of insulin bolused. As the rest of the variables followed in this survey, this was done everyday for a time period of three weeks.

The second part of this study was obtained via the Dexcom sharing feature. The Dexcom reports include data from each day of the week and show their glucose levels at five minute intervals to maximize accuracy. Participants sent me the weekly reports via email from their Dexcom Clarity app. These results from the data trends were then compared to their stress levels for a specific day. In order to account for the confounding variables: physical activity and diet, which may skew a patient's blood sugar to become hyperglycemic, hypoglycemic, or back to a normal state from previously being high or low, the acquired data was plotted on an xy line graph in which days were the independent variable and average glucose readings were the dependent variables. Symbols then indicated when exercise was performed, which tends to lower blood sugar, and when carbohydrates were consumed, which tends to cause a spike in blood sugar. The graphs were acquired from the overlay section of the Dexcom reports which show, as the name suggests, an overlay of lines for all 7 days of the week on a singular line graph displaying their glucose trend. 
Each day was then highlighted in a different bright color and pattern to differentiate between them. The trends from each day were then compared to their daily stress levels, which were completed and submitted through the Google Document via email. This process was repeated for each participant, $\mathrm{A}-\mathrm{V}$, since every person had extremely wideranging results. If the hypothesis was correct and supported by the data, then on days when these type one diabetics were highly stressed due to academics, their glucose levels would reflect this by also being high, and vice-versa, when stress levels were low, glucose level would be on the lower normal spectrum, if exercise and carb and insulin intake did not disrupt it.

\section{Results}

The survey yielded results from exactly 22 type one diabetic teenagers between the ages of 14 and 18 from diabetes support groups across the nation. The mean and median scores of all of the participants' data on their academic stress levels compared to their glycemic levels, is shown in Figures 1 and 2. The data was separated into three groups, low, medium, and high with low representing stress levels between 1 and 3, medium representing stress levels between 4 and 6, and high representing stress levels between 7 and 10. As shown in Figure 1 below, with low stress, participants had a mean of about 2.40 for stress levels and 132.41 for glycemic levels. With medium stress, the mean stress level was about 4.85 and the mean glycemic level was about 150.51. Lastly, with high stress, the mean was about 8.03 for stress and 186.13 for glucose.

\section{Stress Level Glycemic Levels}

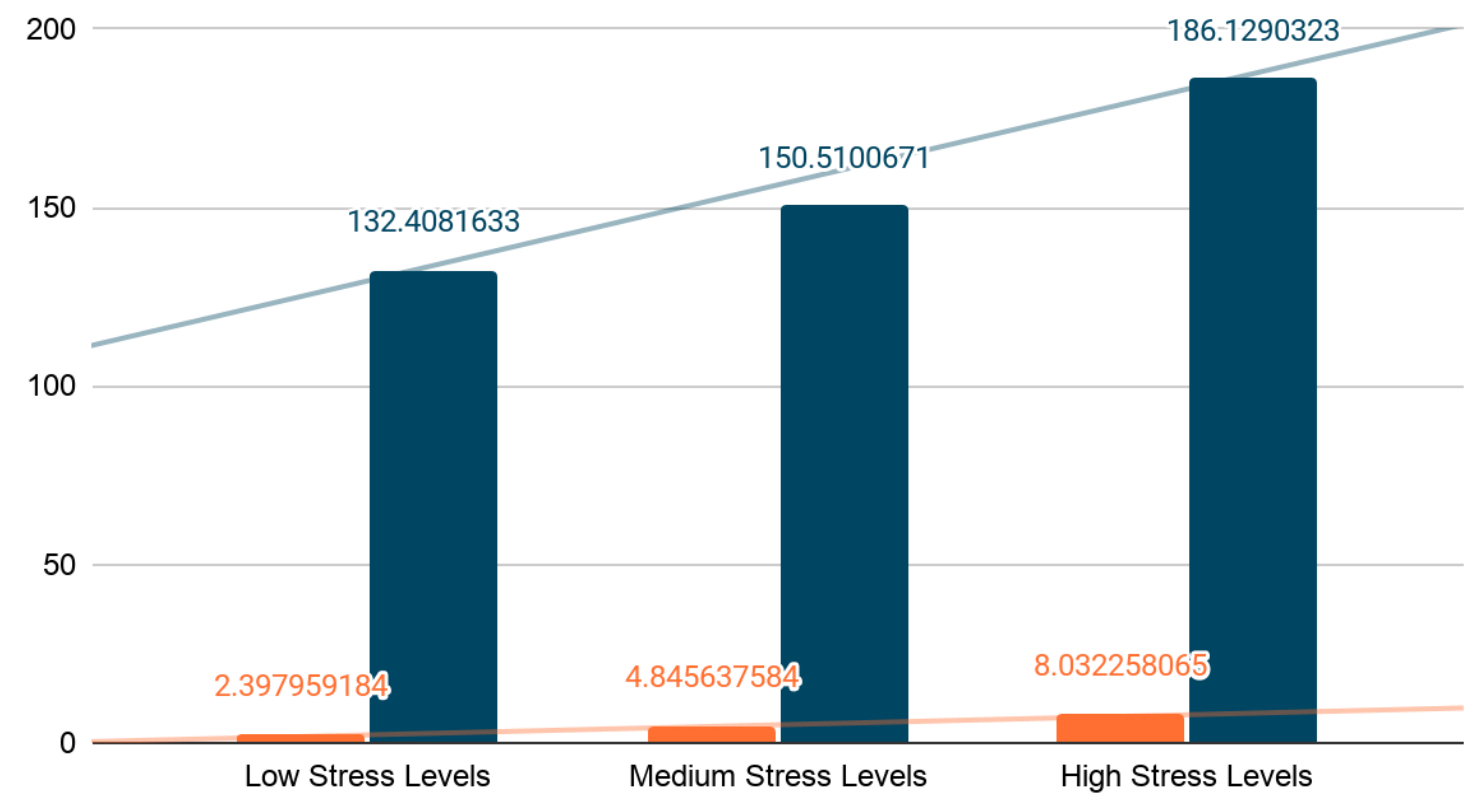

Figure 1. Positive correlation between Stress Level means and Glycemic Level means.

While Figure 1 depicts the mean, Fifure 2 depicts the median values for the data. As shown, the low stress category had a median value of 3 for stress and 123 for glucose; medium had 5 for stress and 151 for glucose; and high had 8 for stress and 188 for glucose. By just looking at both graphs of mean and median, there is a visible increase in both 
stress and glycemic levels between all three categories and is displayed on the graphs with the aid of a trendline connecting the data sets together

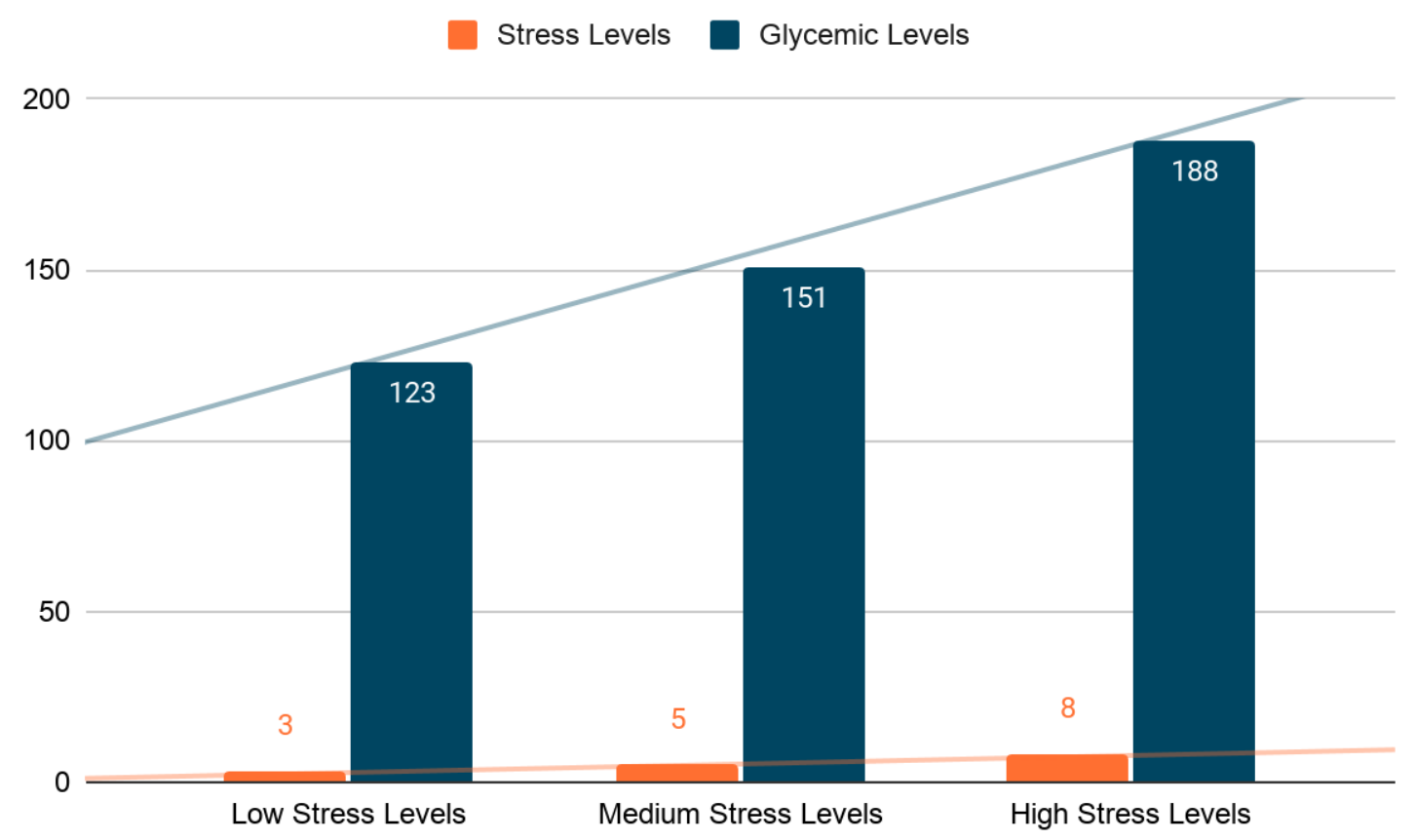

Figure 2. Positive correlation between Stress Level meians and Glycemic Level medians.

Table 1 is used to show the $\mathrm{p}$ and $\mathrm{r}$ values of the means of the data. In the first three rows of the chart, the mean stress and glucose levels are displayed per category of stress, high, medium, and low. These values were already previously mentioned in Figures 1 and 2 with their exact numerical values. These 6 values were used to calculate the $\mathrm{p}$ and $\mathrm{r}$ values which are shown in the bottom two rows of the chart. The $\mathrm{r}$ value of the data set when calculated was 0.9939004022 , indicating a strong positive correlation, and the $p$ value when calculated was $>0.001$, indicating that the data is statistically significant.

Table 1. Statistical Significance and Strength of Correlation of Mean Stress Values

\begin{tabular}{|l|l|l|}
\hline & Stress Level & Glucose Average \\
\hline Low Stress & 2.397959184 & 132.4081633 \\
\hline Medium Stress & 4.845637584 & 150.5100671 \\
\hline High Stress & 8.032258065 & 186.1290323 \\
\hline P- value & $>0.001$ & \\
\hline R- value & 0.9939004022 & \\
\hline
\end{tabular}

Figure 3 depicts a scatterplot of every day recorded by every participant in the study. It shows their glycemic levels in relation to their stress levels. We see a clear upwards trend, made more defined by the black linear line of 
best fit. This shows a positive correlation in which as stress levels increase, so do glucose levels in teenage type one diabetics.

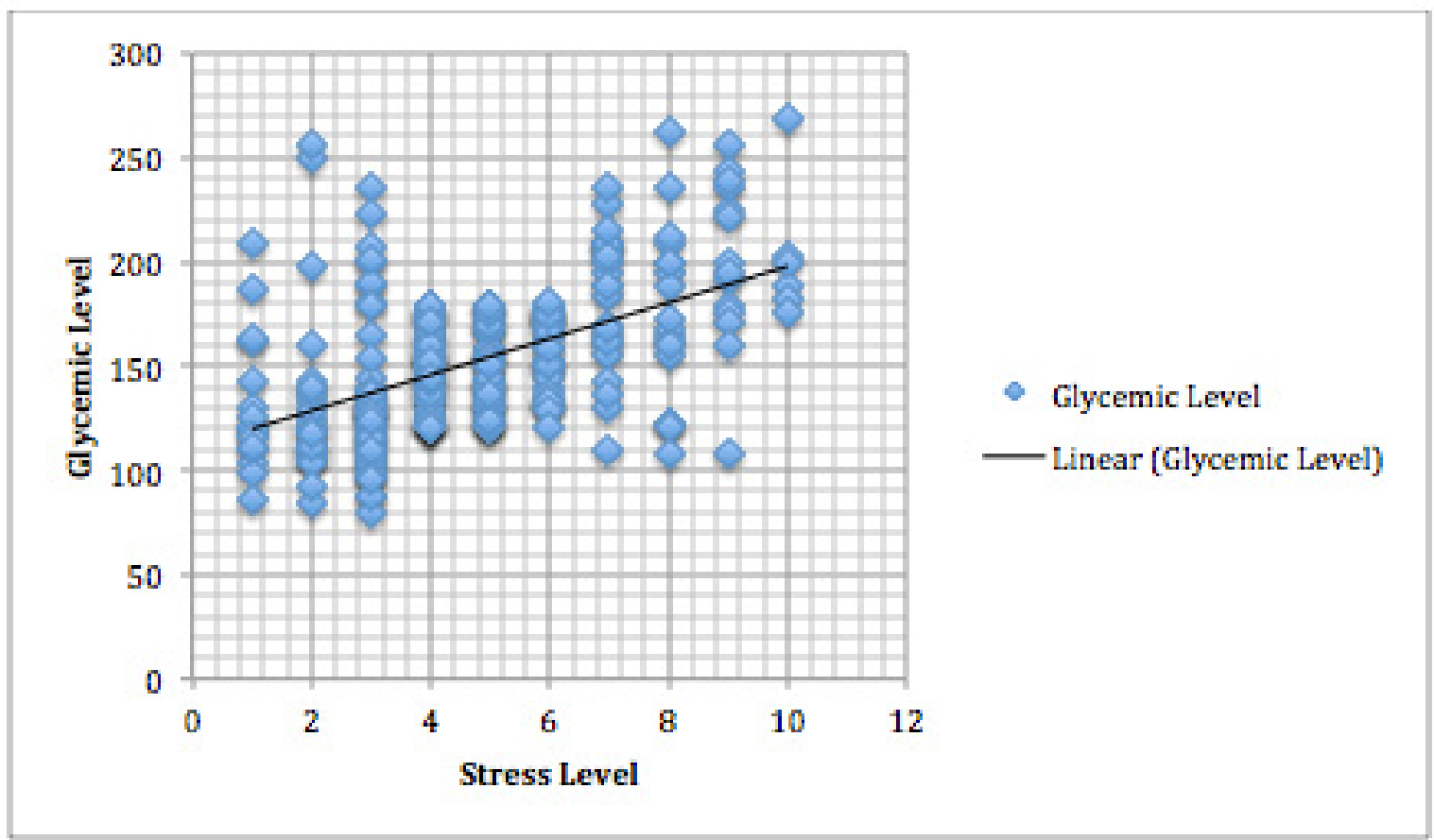

Figure 3. Scatterplot of every value assessed in the study showing a positive correlation between Stress Level and Glycemic Level.

Table 2 is used to show the $\mathrm{p}$ and $\mathrm{r}$ values of the entire data set combined. The $\mathrm{p}$ value when calculated was $>0.001$, indicating that the data is statistically significant. The R-value of the data set in total when calculated was 0.9939004022 , indicating a strong positive correlation.

Table 2. Statistical Significance and Strength of Correlatiom of Entire Data Set

\begin{tabular}{|l|l|l|}
\hline & Stress Level & Glucose Average \\
\hline P-value & $>0.001$ & \\
\hline R- value & 0.912900488 & \\
\hline
\end{tabular}

Figures 4 and Figure 5 both relate to the same participant, Participant "L." Figure 4 is used to display one of the three weeks of data provided by Participant "L." Their average glycemic levels per day are graphed in addition to their selfreported daily stress score. On Monday, their glucose was about 180 with a stress level of 8, on Tuesday they had a glucose average of around 130 with a stress level score of 4, Wednesday was about 110 for glucose and 2 for stress, Thursday was around 140 for glucose and 5 for stress, Friday they were approximately 230 on average and had a stress score of 9, Saturday showed about 170 for glucose and 7 for stress, and finally, Sunday displays a mean score of about 140 as far as glucose and 5 as far as stress. For this chart specifically, blue pertains to glucose level values which are shown on the left side of the graph while orange pertains to stress levels and its values are shown on the right side of the graph. 
Figure 5, on the other hand, shows more in depth data of just one of Participant "L"'s recorded days, day number 7. It is important to remember that this specific graph was auto-generated by the Dexcom application in order to get accurate and continuous results in intervals of 5 minutes. The default settings of this graph make the target glucose range during the day (from $6 \mathrm{am}$ to $10 \mathrm{pm}$ ) between 80 and 180 and during the nighttime (from 10pm to 6am) the target range shifts to be in between 70 and 150. Using Dexcom's default settings, the graph shows that the participant was mostly steady for the majority of the day except for 3 high blood sugars at 12 am, 12 pm, and 12 am once again on the following day. They also had one low blood sugar at around 3:30 pm according to the graph. The graph also depicts two times of carb intake at about 9:45 am and 8:15 pm which were also followed by insulin intake according to their answers on the survey not depicted here.

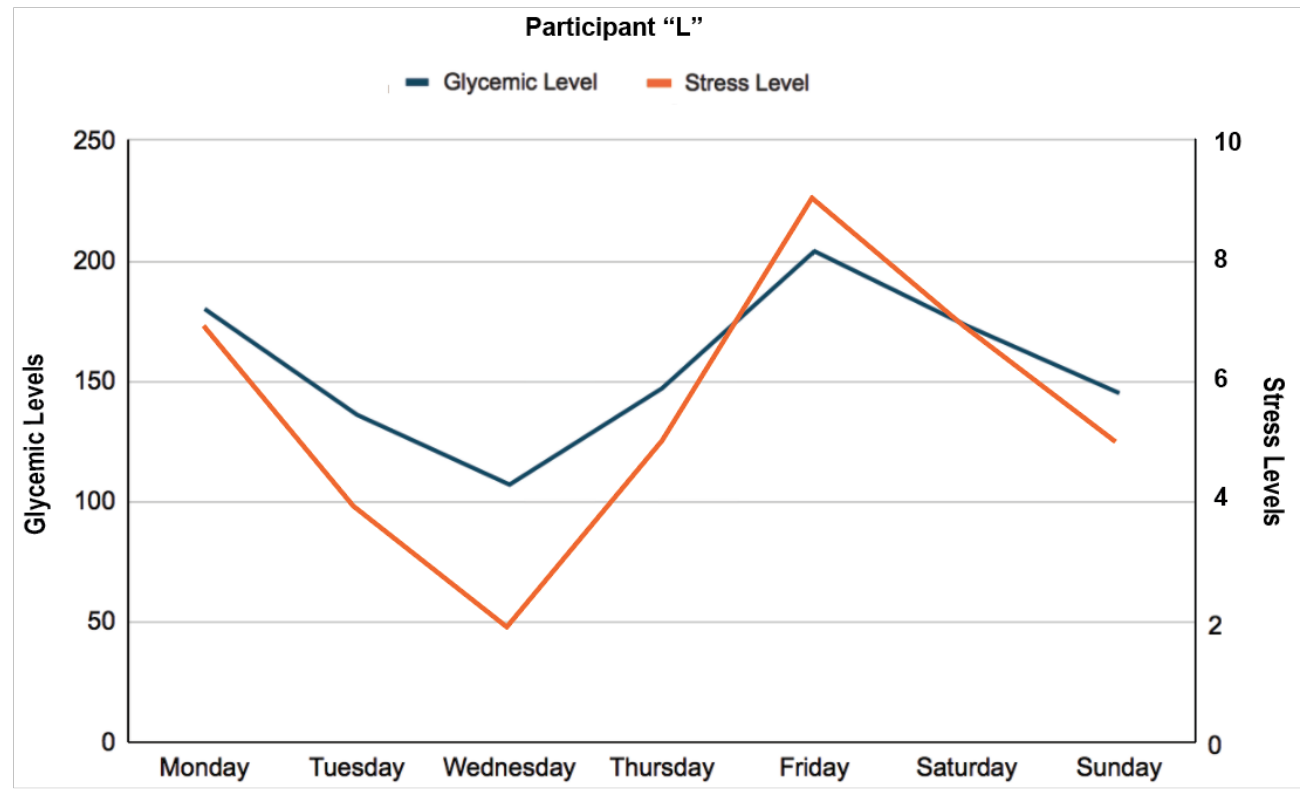

Figure 4. Weeklong results for participant with most statistically significant data.

Participant "L": Day \#7

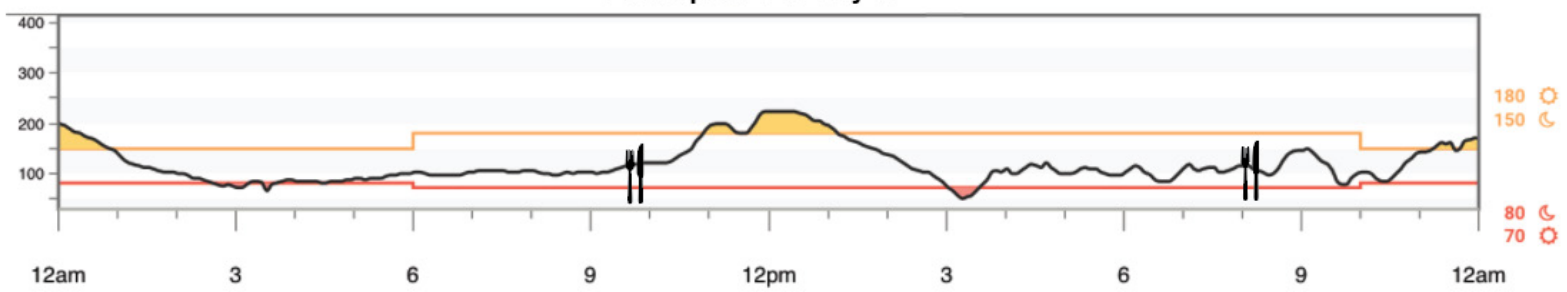

Figure 5. Daily results for participant with most statistically significant data.

Figures 6 and 7 represent the same values as Figures 4 and 5 do, but for another participant, Participant "F." Figure 5, much like Figure 3, depicts one of the three weeks of data provided by Participant " $F$ " and their average glucose levels 
per day compared to their self-evaluated daily stress score values. According to this graph, on Monday, their glucose was at an average of 207 and stress level of 2, on Tuesday their glucose was an average of 174 and their stress was at level 3, Wednesday they had a mean glucose of 181 and stress score of 1, Thursday showed a mean of 160 for glucose and 1 for stress, Friday was at an average of 199 for glucose and 2 for stress, Saturday their glucose mean was 133 and stress was at level 3, and lastly, on Sunday they had an average of 145 for their glucose and a level of 3 for stress. This chart as well follows the key in which blue pertains to glucose level values that are shown on the left side of the graph while orange pertains to stress levels and its values are shown on the right side of the graph.

Figure 6. Weeklong results for participant with most confounding variables.

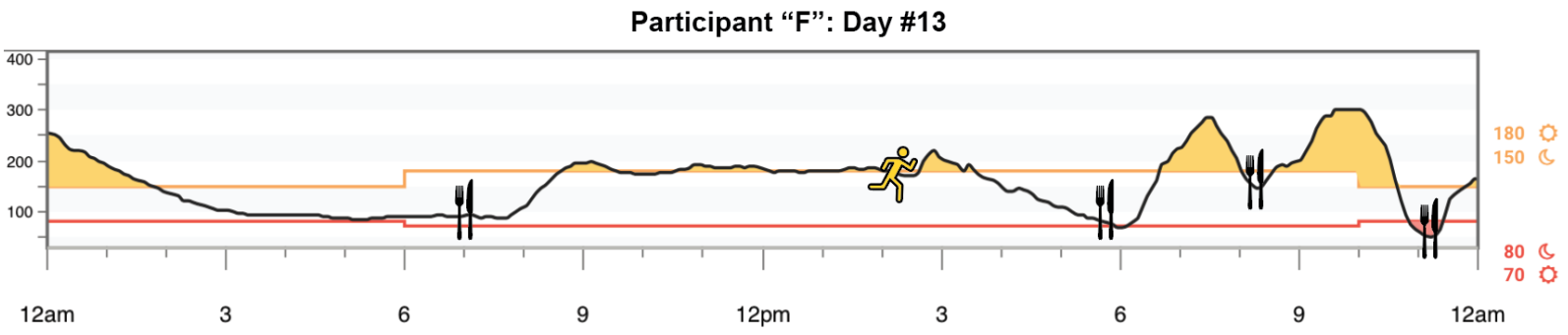

Figure 7. Daily results for participant with most confounding variables.

\section{Conclusion}

Figures 1 and 2 clearly show a clear positive correlation between the two variables. They show that as stress levels caused by academics increase, so do glycemic levels in type one diabetic patients. We can see this positive trend since the mean and median of both stress levels and glycemic levels increase as the categories of blood sugar increase from low to medium to high. Table 1 reinforces the conclusions drawn from Figures 1 and 2 . It shows that the r-value of all the means in the data set is about 0.994 . This numerical value proves a significantly strong correlation between stress and glucose since it is only about 0.1 points off from being a perfect positive 1 . The p-value reinforces this principle as well by equaling to approximately 0.005 . Since the number is so small, it conveys that the null hypothesis is rejected and the results found are statistically significant.

Figure 3 is much like 1 and 2 because it shows the clear positive correlation between stress and glycemic levels in which they both increase simultaneously. Although there appears to be many outliers, there are so many data values, that they barely effect the overall correlation. In Table 2 we are able to see the numerical values for P and $\mathrm{R}$ values which indicate statistical significance. Since r-value was so high, about 0.913 , it means that there is a strong positive correlation throughout the entirety of the data .Like in Table 1, p-value conveys that the null hypothesis is rejected and the results found are statistically significant since the value is so small.

Additionally, Figure 4 also supports the same claim, but showing a more specific example from just one participant, "Participant L." This participant shows an excellent example of the positive correlation discussed. They also had minimal confounding variables that may skew their data meaning that the results were as close to accurate as possible. In this table, this participants' weekly glucose trends are compared to their weekly stress level trends side by side. Both lines follow each other very closely and have a very similar shape and path to them, once again supporting the idea of a positive correlation. Table 5 relates to Participant $\mathrm{L}$ as well, but dives into one specific day of the 3 weeks monitored to give an example of the confounding variables. The icons placed on the line graph coincide with the participants' time of insulin bolus as well as time of exercise or any sort of physical activity and the color of the icon 
correlates to intensity. By analyzing this set of data, we can see that these two possible confounding variables had little to no effect on the person's glycemic level, making them a prime example from which to draw conclusions. This is known because the p-value for this specific participant is 0.9952783653 , only about 0.1 off from a perfect positive 1 and displays an extremely strong positive correlation.

Tables 6 and 7 correlate to another participant, Participant F. This specific participant shows no correlation at all since their data points are scattered and non-uniform. Their values ultimately skewed the data slightly, but since the correlation was so strong, we were able to still see the overall positive correlation although this participant's data did increase the standard deviation heavily. Table 6 individually shows their glucose trends compared to their weekly stress level trends just like Table 3 does for Participant L. These trends, when compared, do not seem to have any sort of correlation to each other since they have very different trends when compared to each other. They seemed to have had very high daily glucose averages, but also had extremely low stress levels most of the time. However, when looking at Table 7 which displays the glucose data of one specific day of the 3 weeks monitored as well as icons for insulin dosages and physical activity, just like in Table 5, there is reasoning behind this nonuniformity. Participant "L" seemed to have very high carb intake along with small values of the units of insulin bolused to cover them along with no exercise documented. Everytime a "carby" meal was consumed, a large spike in glucose levels was observed shortly after skewing their daily averages to be extremely high in the 200 range.

This participant as well as another one, Participant " $\mathrm{H}$ ", both were considered outliers and slightly skewed the overall results. Since the data still showed an extremely strong positive correlation even with them included, they were still included in reaching a conclusion as well as for ethical reasons. This proves just how strong the correlation is and there is much evidence supporting the reason this skew might have occurred in their specific data sets in the confounding variables section.

While the data provided supports the conclusion that there is a positive correlation between type one diabetic teenagers' academic stress levels and their glycemic levels, this does not necessarily mean that one causes the other. There are too many confounding variables to say that one variable directly causes the other one to shift. Additionally, all that is known is a correlation between the two, not an actual causation.

\section{Limitations, Implications, and Future Research}

Given the positive correlation between academic stress levels and glycemic levels in teenage type one diabetics, there should be further research into treatments that address the needs of these patients. This study shows stress is having some negative impact on glucose levels of type one diabetics between the ages of 14 and 18 and this can lead to a high A1C level or put patients at highest risk for other life threatening conditions such as kidney and heart failure among many other consequences. Teachers in school should welcome their students, specifically T1D students to be able to talk to them about their personal struggle with the workload and overall mental health and be offered extended time on assignments. Many patients do have this available with their 504 plan in school, but if they don't, they should still have the option open to them.

These findings do have clear limitations such as the wide range of insulin dosages patients take, as well as the imprecise nature of self-reported diet and exercise. The social isolation guidelines in place due to Covid-19 are also a limitation to consider since they precluded in person studies and interviews. Finally, the study had purposeful delimitations of type one diabetics but that does leave a gap for further research on a wider range of non-diabetic patients or even in gender specific cohorts.

\section{Acknowledgments}

I would first like to express my sincere appreciation to my AP Capstone Research teacher, Mrs. Sierra, for her dedication, passion, and constant support throughout every aspect of this process not only to me, but every one of her 
students. I would also like to thank everyone who participated in this research project as it would not have been possible without their cooperation as well as commitment to it. Finally, I would like to thank my parents and closest friends and peers who have supported me from the very beginging and motivated me to keep going. Without any of these individuals and their guidance, I would not be where I am today; thank you once again.

\section{References}

Ahola , A., Forsblom , C., Harjutsalo, V., \& Groop, P.-H. (2020, July 18). Perceived Stress and Adherence to the Dietary Recommendations and Blood Glucose Levels in Type 1 Diabetes. Journal of diabetes research. https://pubmed.ncbi.nlm.nih.gov/32733965/.

American Diabetes Association (ADA) Statistics About Diabetes. Statistics About Diabetes(2018). https://www.diabetes.org/resources/statistics/statistics-about-diabetes.

Attari, A., Sartippour, M., Amini, M., \& Haghighi, S. (2006, July). Effect of stress management training on glycemic control in patients with type 1 diabetes. Diabetes research and clinical practice. https://pubmed.ncbi.nlm.nih.gov/16476501/.

Bending, D., Cooke, A., \& Zaccone, P. (2012, June). Inflammation and type one diabetes. International immunology. https://pubmed.ncbi.nlm.nih.gov/22447815/.

Dexcom, Inc. (2021). Welcome to Dexcom CLARITY, your diabetes management application.Dexcom CLARITY. https://clarity.dexcom.com/.

Ferreira, L., Teixeira-de-Lemos, E., Pinto, F., Parada, B., Mega, C., Vala, H., .. Reis, F. (2010, June 21). Effects of Sitagliptin Treatment on Dysmetabolism, Inflammation, and Oxidative Stress in an Animal Model of Type 2 Diabetes (ZDF Rat). Mediators of Inflammation. https://www.hindawi.com/journals/mi/2010/592760/.

Goetsch, V. L., Wiebe, D. J., Veltum, L. G., \& Dorsten, B. van. (1990, June 6). Stress and blood glucose in Type II diabetes mellitus. Behaviour Research and Therapy. https://www.sciencedirect.com/science/article/abs/pii/000579679090140E.

Radahmadi , M., Shadan, F., Karimian, S., Shahab-e-din Sadr, S., \& Nasimi, A. (2006, February 21). Effects of stress on exacerbation of diabetes mellitus, serum glucose and cortisol levels and body weight in rats. Pathophysiology : the official journal of the International Society for Pathophysiology. https://pubmed.ncbi.nlm.nih.gov/16102950/.

Surwit, R. S., Schneider, M. S., \& Feinglos, M. N. (1992, October 1). Stress and Diabetes Mellitus. Diabetes Care. https://care.diabetesjournals.org/content/15/10/1413.

Trapp , M., Costa Valle, S., Gomes Pöppl, A., Fernandes Chittó , A. L., Kucharski , L. C., \& Silveira Martins Da Silva , R. (2018, June 1). Insulin-like receptors and carbohydrate metabolism in gills of the euryhaline crab Neohelice granulata: Effects of osmotic stress. General and comparative endocrinology. https://pubmed.ncbi.nlm.nih.gov/29548758/. 